\title{
Neutron Through-Thickness Stress Measurements in Two-Phase Coatings with High Spatial Resolution
}

\author{
V. Luzin ${ }^{1, a^{*}}$ and D. Fraser ${ }^{2, b}$ \\ ${ }^{1}$ Australian Nuclear Science and Technology Organisation, Lucas Heights, NSW, 2232 Australia \\ ${ }^{2}$ Commonwealth Scientific and Industrial Research Organisation, Clayton, Melbourne, VIC, \\ 3168, Australia \\ avladimir.luzin@ansto.gov.au, ${ }^{\mathrm{b}}$ Darren.Fraser@csiro.au
}

Keywords: Residual Stress, Coatings, Cold Spray

\begin{abstract}
Neutron diffraction residual stress profiling of sprayed coatings with high spatial resolution is a difficult task. Normally, only for single-phase materials $0.1-0.2 \mathrm{~mm}$ resolution can be achieved. Stress measurements in two-phase or multi-phase coatings are an even more formidable experimental task due to the necessity of measuring all phases with lower than $100 \%$ volume fractions and the necessity to resolve the $\mathrm{d}_{0}$ problem in a more complex way than the for single-phase coating systems.

The results of through-thickness residual stress profiling neutron diffraction experiments are reported on a selected two-phase, metal-metal composite coating, deposited by the cold spray technique. With both phase strains measured and complemented by additional information provided by other characterisations, the full stress state was reconstructed. Macro- and microstresses were separated allowing interpretation of the experimental data in terms of macro- and micro-mechanics. It also allowed us to make conclusions about the thermal mechanisms of macro- and micro-stress formation, as well as connection of these mechanisms to spraying parameters.
\end{abstract}

\section{Introduction}

A range of spray technologies are in use now for the purpose of surface enhancement and are frequently employed to produce coatings on the surface of numerous engineering components. Coatings of many different materials are produced by various spray techniques for numerous applications, such as wear resistance, corrosion protection, insulation, etc., and usually the spraying conditions and parameters are optimized for the purpose. One of the parameters to consider in such optimisation is the residual stress which is formed due to high temperatures or/and high kinetic energies associated with the spraying process, as well as mismatches in the substrate and coating material properties. Since the residual stress, which can negatively influence the coating's mechanical integrity or functional performance, stress control and mitigation are usually an integral part of the technology.

Neutron stress measurement in thick and thin coatings has proven to be a useful method since it is non-destructive, it can provide the required high resolution (down to $0.2 \mathrm{~mm}$ ), it does not require special sample preparation (e.g. cutting and polishing, as for X-rays), measurement can be done in a reasonable time (minutes per datum) and with high accuracy (uncertainty can be better than $5 \mathrm{MPa}$ ). However, some cases remain challenging, e.g. $0.1 \mathrm{~mm}$ thick coatings, and another challenge is stress measurement in two-phase coatings, especially when the volume fraction of one of the phases is small.

In this work we report an experimental study of the residual stress analysis in the two-phase (metal-metal) coating-on-substrate system produced by the cold-spray technique. 


\section{Specifics of the stress measurements in two-phase coatings}

Details of stress measurements of two-phase systems were outlined in [1], only a few key points are highlighted here. Macrostress profiling can be achieved through measurements of both phases and averaging phase stresses with the corresponding volume fractions, which can be found in a separate (e.g. neutron powder diffraction) experiment. As in a single-phase coating experiment, measurements of the strains in the in-plane and normal directions are required. There is no need for knowing exact $\mathrm{d} 0$ values if only the macrostress is of interest. Regarding the microstress, if no stress-free, $\mathrm{d}_{0}$-powders are available, only the deviatoric part of the microstress tensor can be found. If, however, the $\mathrm{d}_{0}$-powders (or at least one powder) are available, the hydrostatic part can be derived from the analysis of strain data.

\section{Samples}

The powder for spraying has chemical composition typical for the lean duplex stainless steels, though with somewhat high Mn content, so that the sprayed material has both fcc $(\gamma-\mathrm{Fe})$ and bcc $(\alpha-\mathrm{Fe})$ iron. The chemical and phase composition was developed for a special (undisclosed) application requiring high temperature oxidation resistance and strength. Size distribution of the multi-disperse spherical powder particles, with diameter being within the range 5 to $50 \mu \mathrm{m}$ (Fig. 1 ), was quantified resulting in the average particle size of approximately $23 \mu \mathrm{m}$. The details of the chemical compositions are given in Table 1.
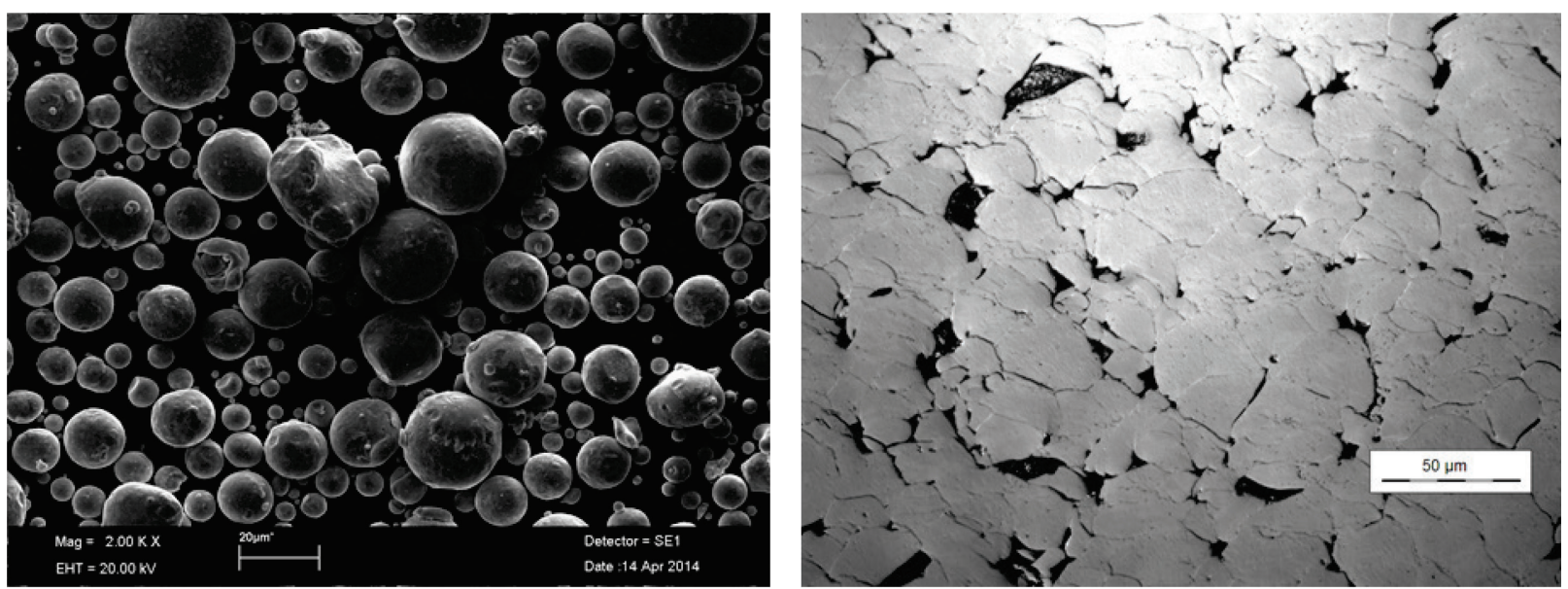

Fig. 1: Morphology and sizes of particles of the alloy powder (left) and microstructure of the sprayed material, sample \#1 (right). Dark areas of the microstructure correspond to porosity.

Table 1. Chemical composition of the initial powders, wt.\%.

\begin{tabular}{|c|c|c|c|c|c|c|c|}
\hline & $\mathrm{C}$ & $\mathrm{Mn}$ & $\mathrm{Cr}$ & $\mathrm{Mo}$ & $\mathrm{Ni}$ & $\mathrm{Co}$ & $\mathrm{Si}$ \\
\hline duplex stainless steel & $<0.03$ & 8.7 & 21.7 & $<0.1$ & 4.4 & 4.2 & 0.37 \\
\hline
\end{tabular}

To produce samples for the study, the powder was cold sprayed using the CSIRO's high pressure system, CGT Kinetiks 4000. While the gas (nozzle) temperature and pressure were fixed at $800^{\circ} \mathrm{C}$ and 35 bars respectively, some spray parameters were varied accordingly to Table 2. Relatively high temperature for the cold-spray gas technique was required to provide deposition conditions for the powder particles, as the particle size was relatively large for the cold spray. The combinations of spraying parameters were chosen in such a way that under assumption of constant deposition efficiency the expected thickness of coatings should be 3.0 $\mathrm{mm}$. It was evident that for different regimes (sets of spray parameters, Table 2) the efficiency was not the same resulting in coatings of different thicknesses. All coatings were sprayed on 
similar sized substrate patches, i.e. squares $40 \times 40 \mathrm{~mm}^{2}$ and $6.2 \mathrm{~mm}$ in thickness, machined from austenitic stainless steel 316.

Table 2. Sample spraying parameters.

\begin{tabular}{|l|l|c|c|c|c|}
\hline Sample ID & powder & $\begin{array}{l}\text { Feed rate, } \\
{[\mathrm{kg} / \mathrm{hour}]}\end{array}$ & $\begin{array}{l}\text { Traverse speed, } \\
{[\mathrm{mm} / \mathrm{s}]}\end{array}$ & Passes & $\begin{array}{l}\text { Thickness, } \\
{[\mathrm{mm}]}\end{array}$ \\
\hline$\# 1$ & duplex steel & 1 & 300 & 60 & 3.1 \\
\hline$\# 2$ & duplex steel & 2 & 300 & 30 & 2.7 \\
\hline$\# 3$ & duplex steel & 1 & 100 & 20 & 1.5 \\
\hline
\end{tabular}

Elastic properties and density evaluation

For the Young's modulus measurements, rectangular specimens were extracted from the bulk of the coating with approximate dimensions $38 \times 5 \times 2 \mathrm{~mm}^{3}$. The Young's modulus was determined using the Impulse Excitation Technique (IET) according to the ASTM standard E1876 through acoustic measurements of the normal frequency. For the given sample dimensions the accuracy of this method was generally better than $1 \%$. The same samples with highly accurate dimensions were used to evaluate their density through volume calculation and sample weighing. The results of the evaluations are reported in Table 3.

\section{Phase composition measurements}

The full neutron diffraction patterns were measured using the same bar samples for the evaluation of phase composition. Being a volumetric technique, neutron diffraction provided highly reliable bulk averaged volume fraction for bcc vs fcc. The diffraction patterns were measured over an angular range of $10-160^{\circ}$ at a wavelength of $1.622 \AA$ using the high resolution powder diffractometer ECHIDNA at the ANSTO OPAL research reactor [2]. The volume fractions of the phases were determined using the GSAS Rietveld refinement software [3] with the EXPGUI interface [4].The results of the determinations are reported in Table 3.

\section{Neutron residual stress measurements}

Neutron diffraction residual stresses measurements have been carried out using the stress diffractometer KOWARI at the ANSTO OPAL research reactor [5]. For through-thickness stress measurements a gauge volume with dimensions $0.5 \times 0.5 \times 20 \mathrm{~mm}^{3}$ was used. The gauge volume was small enough to provide the necessary through-thickness resolution while producing a count rate sufficiently high for determining strain measurement in each phase with statistical uncertainty better than $5 \times 10^{-5}$ within a reasonable measurement time. The exposure times were $\sim 1$ minute per position for the measurements in the substrate material and 5 minutes per point for the coating material due to significant effect of peak broadening. All measurements were done with a neutron beam wavelength of $1.54 \AA$ providing approximately $90^{\circ}$-geometry for the two reflections that investigated, i.e. $\gamma-\mathrm{Fe}(311)$ and $\alpha-\mathrm{Fe}(211)$ with the diffraction angles being $90^{\circ}$ and $82^{\circ}$ respectively.

The strain measurements were carried in many through-thickness positions covering the entire sample thickness. The $0.5 \mathrm{~mm}$ spacing between points was chosen to be proportional to the overall thickness of $\sim 9 \mathrm{~mm}$ and gauge volume size of $0.5 \mathrm{~mm}$. Notwithstanding the equi-biaxial stress state most likely to be expected, two in-plane directions and one normal direction were measured in order to reconstruct two in-plane stress principal components under the assumption of plane (macro-) stress condition.

A "substrate only" sample was measured with the same measurement protocol confirming the absence of any pre-existing stress distribution in the substrate material (e.g., from the production stage). 
The experimentally determined phase d-spacing data were treated according to the stress reconstruction procedure [1] resulting in the macrostress through-thickness profiles (Fig. 3).

The macrostress, determined through the rule-of-mixture, $\sigma^{M}=f_{\alpha} \sigma_{\alpha}^{t}+f_{\gamma} \sigma_{\gamma}^{t}$, where $\sigma_{\alpha}^{t}$ and $\sigma_{\gamma}^{t}$ are total phase stresses, was further treated within an empirical stress formation model, the progressive layer deposition model by Tsui \& Clyne [6]. The thermal mismatch term, $\Delta \varepsilon=$ $\Delta \alpha \Delta \mathrm{T}$, due to the difference $\Delta \alpha$ between the coefficients of thermal expansion (CTE) of the substrate material (SS316) and coating material ( $\alpha-\mathrm{Fe} / \gamma-\mathrm{Fe}$ composite), was found (as a best fit) to be the same for all three samples, $\Delta \varepsilon=-156 \pm 5 \mu$ strain. This corresponds to a temperature drop after spraying $\Delta \mathrm{T}_{1} \sim 380-400^{\circ} \mathrm{C}$, making some assumption about the CTE of the coating phases, $\alpha$-Fe and $\gamma$-Fe. The deposition stress was thus sample dependent and reported in Table 3 .

The deviatoric part of the microstress tensor was also derived and shown in Fig. 4. Since it was impossible to obtain $\mathrm{d}_{0}$-powders for any of the coating constituents, the hydrostatic part of the microstress was not determined experimentally. Instead, it was evaluated in a model approach, e.g. within Hashin-Shtrikman bounds theory [7], knowing that the hydrostatic part can only be generated thermally, taking into account the experimentally known phase compositions, assuming typical CTEs of $\alpha-\mathrm{Fe}$ and $\gamma$-Fe and temperature drop $\Delta \mathrm{T}_{2} \sim 700^{\circ} \mathrm{C}$. The results of the total stress evaluation are shown in Fig. 4.

Table 3. Sample characterization: elastic properties, phase composition and stress analysis.

\begin{tabular}{|l|l|l|c|c|}
\hline $\begin{array}{l}\text { Sample } \\
\text { ID }\end{array}$ & $\begin{array}{l}\text { Young's } \\
\text { modulus, [GPa] }\end{array}$ & $\begin{array}{l}\text { Density, } \\
{\left[\mathrm{g} / \mathrm{mm}^{3}\right]}\end{array}$ & $\begin{array}{l}\text { Volume fractions, } \\
\text { [aus/ferr] }\end{array}$ & $\begin{array}{l}\text { Deposition stress, } \\
{[\mathrm{MPa}]}\end{array}$ \\
\hline$\# 1$ & $129.6 \pm 0.5$ & $7.259 \pm 0.034$ & $0.44 / 0.56$ & $+11 \pm 20$ \\
\hline$\# 2$ & $125.4 \pm 0.7$ & $7.237 \pm 0.045$ & $0.46 / 0.54$ & $+53 \pm 20$ \\
\hline$\# 3$ & $115 \pm 2.5$ & $6.858 \pm 0.15$ & $0.53 / 0.47$ & $+99 \pm 20$ \\
\hline
\end{tabular}

\section{Discussion}

Macrostress. In all three samples there were two contributions to the macrostress, i.e. the thermal mismatch and the deposition stress. While thermal mismatch generated compressive stress in the coating, the deposition stress is tensile. The fact that the resultant stress is still compressive illustrates the dominant role of the thermal mismatch mechanism. Contrary to expectation of a compressive deposition stress, typical for the cold-spray materials [8], it was tensile, which is more typical for thermal spray techniques like plasma or HVOF (High Velocity Oxi-Fuel). Since the sign of the deposition stress is determined by a balance between the peening mechanism (compressive stress) and the quenching mechanism (tensile stress), our results suggest a much more significant role of quenching than peening, thus making the given regime of the cold-spray system being more reminiscent to HVOF.

Among the samples, the deposition stress parameter demonstrated a clear trend being more tensile for sample \#3 and less tensile for sample \#1. Assuming the above discussed concept of the peening/quenching balance, sample \#1 exhibits the most amount of peening that is also reflected in a higher value of the Young's modulus, density and efficiency (accumulated thickness) due to better compaction of splats in the coating. The opposite is valid for sample \#3.

Anti-correlation of the deposition stress and number of passes makes a link to the spray parameters: for sample \#3 with only 20 passes, the localized heat-input must be greater than for the faster moving heat source for sample \#1 with 60 passes, which allows more efficient heat dissipation and, therefore, reducing the role of the thermal/quenching effects.

Microstress. The hydrostatic microstress is clearly thermally generated, due to the difference between CTE of $\alpha$-Fe and $\gamma$-Fe, and it is tensile in $\gamma$-Fe and compressive in $\alpha$-Fe. Since the phase 
composition for the three samples are close, the microstress is approximately the same, approximately $400 \mathrm{MPa}$ in $\gamma$-Fe and $-400 \mathrm{MPa}$ in $\alpha$-Fe.
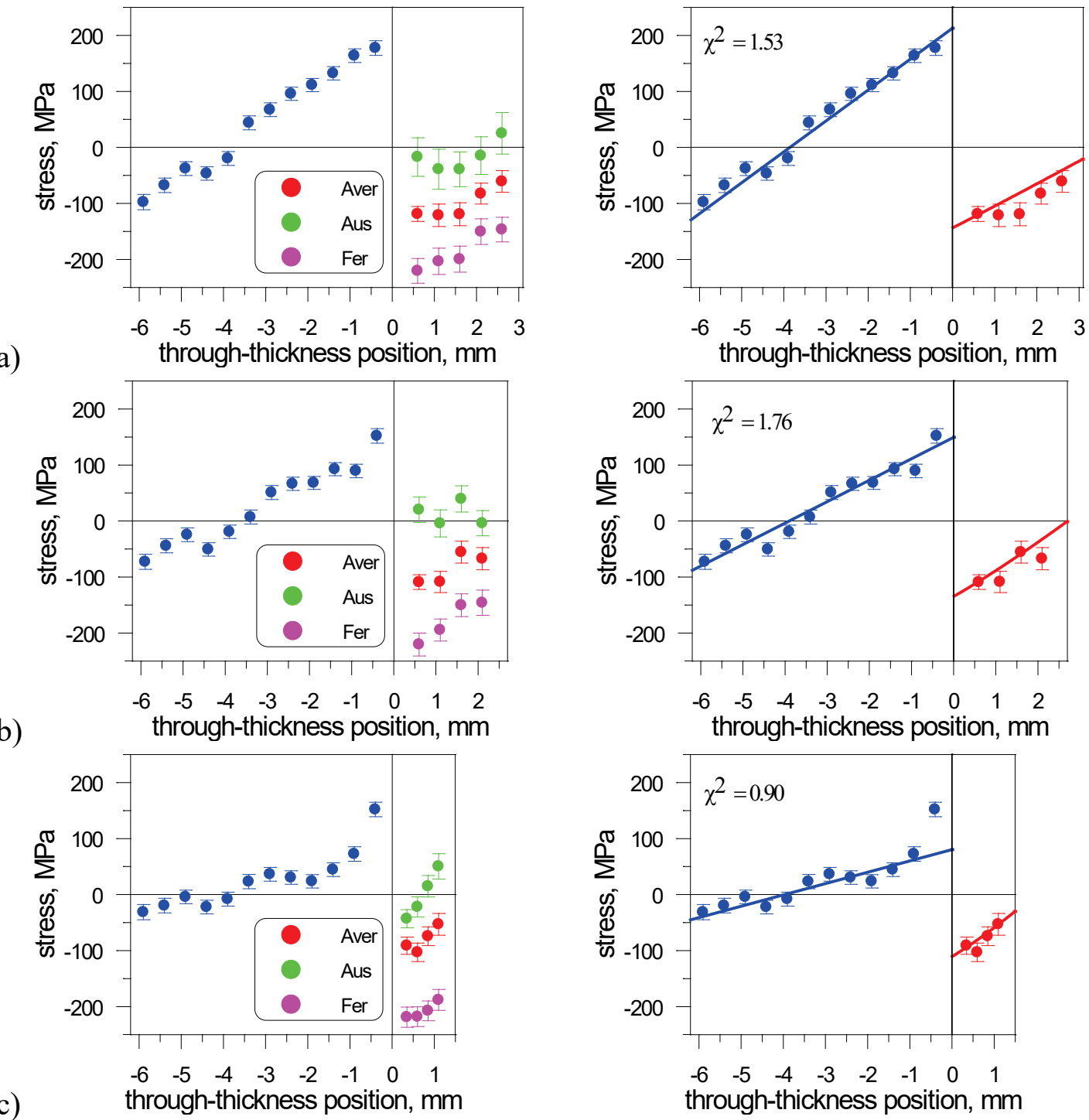

Fig. 3: Experimentally measured phase stresses (left column) and model fit of the macrostress profiles (right column) for three samples \#1, \#2 and \#3, correspondingly a), b) and c). Aus represents the austenite phase and Fer the ferrite phase respectively.

The results show clear stress relaxation for the normal component - a reason for appearance of the deviatoric component. Two micro-mechanical mechanisms can be responsible, (i) oriented microcracking with the crack plane being parallel to the surface and (ii) plastic mismatch which is manifested in a differential amount of plastic deformation in the two phases, more plastic fcc $\gamma$-Fe vs less plastically hard bcc $\alpha$-Fe. Considering the real microstructure (Fig. 1) exhibiting approximately round splats (assuming a small amount of the overall plastic deformation, but most likely large in localized areas) and visible porosity, the first mechanism (i) is most plausible, though with some presence of mechanism (ii). This also can be supported by a correlation between the amount of stress relaxation in the normal direction (largest in \#3) and deterioration of the Young's modulus (largest in \#3), which is also sensitive to microcracking. 

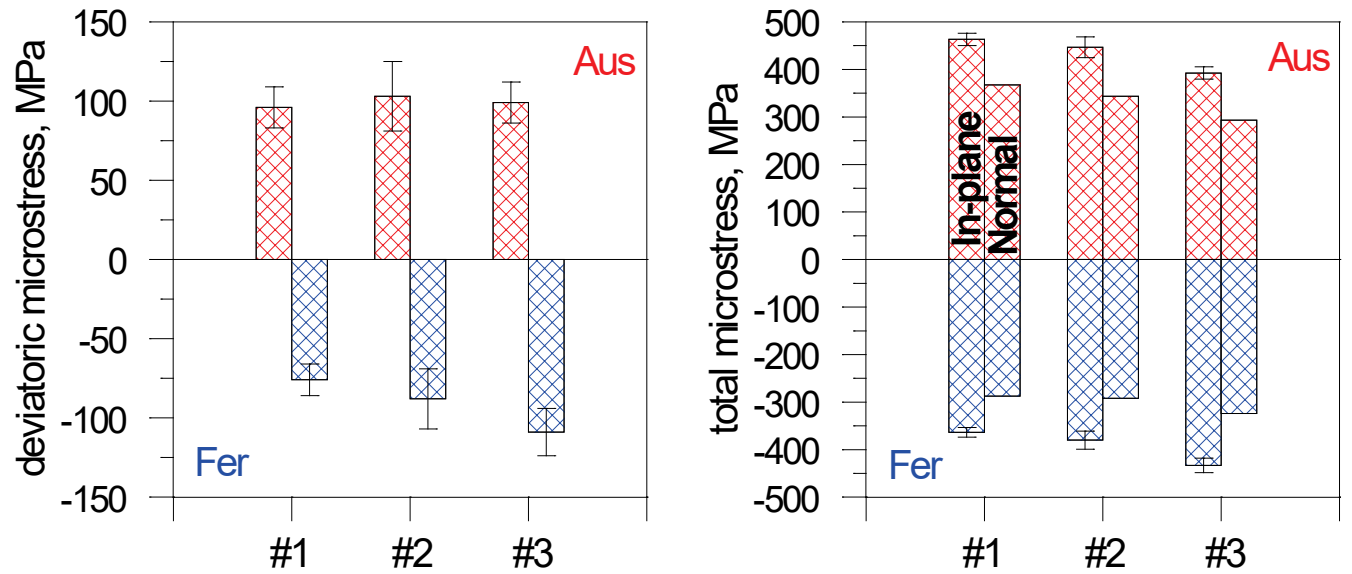

Fig. 4. Deviatoric part of the microstress (left, as measured) and the total microstress (right) obtained by adding the hydrostatic part (calculated). Aus represents the austenite phase and Fer the ferrite phase respectively.

\section{Conclusions}

Three different composite $(\gamma-\mathrm{Fe} / \alpha-\mathrm{Fe})$ coating samples were sprayed by a cold-spray system in a high temperature regime, thus being closer to the thermal spray conditions but without melting. Stresses were measured in both phases with neutron diffraction with high spatial resolution of $0.5 \mathrm{~mm}$. It was sufficient to resolve stress profiles in $1.5-3.1 \mathrm{~mm}$ thick coatings and make quantitative analysis possible. Experimentally determined macrostresses and microstresses were matched with modelling results to reveal the macro- and micro-mechanical mechanisms of stress formation, dominated in both cases by thermal nature.

\section{References}

[1] V. Luzin, J. Matejicek and G.-H. T., Through-thickness Residual Stress Measurement by Neutron Diffraction in Cu+W Plasma Spray Coatings, Mater. Sci. Forum 652 (2010) 50-56. https://doi.org/10.4028/www.scientific.net/MSF.652.50

[2] K.-D. Liss, B. Hunter, M. Hagen, T. Noakes and S. Kennedy, Echidna - the new highresolution powder diffractometer being built at OPAL, Physica B: Condensed Matter 385-386 Part 2 (2006) 1010-1012. https://doi.org/10.1016/j.physb.2006.05.322

[3] A.C. Larson and R.B. Von Dreele, in: Report LAUR 86-748, Los Alamos National Laboratory, 2004.

[4] B. Toby, EXPGUI, a graphical user interface for GSAS, J. Appl. Crystallogr. 34 (2001) 210213. https://doi.org/10.1107/S0021889801002242

[5] O. Kirstein, V. Luzin and U. Garbe, The Strain-Scanning Diffractometer Kowari, Neutron News, 20 (2009) 34-36. https://doi.org/10.1080/10448630903241175

[6] Y.C. Tsui and T.W. Clyne, An analytical model for predicting residual stresses in progressively deposited coatings Planar geometry, Thin Solid Films 306 (1997) 23-33. https://doi.org/10.1016/S0040-6090(97)00199-5

[7] Z. Hashin, The Elastic Moduli of Heterogeneous Materials, J. Appl. Mech. 29 (1962) 143150. https://doi.org/10.1115/1.3636446

[8] V. Luzin, K. Spencer, M. Zhang, N. Matthews, J. Davis and M. Saleh, Residual Stresses in Cold Spray Coatings, in: P. Cavaliere (Ed.) Cold-Spray Coatings - Recent Trends and Future Perspectives, Springer, 2018. https://doi.org/10.1007/978-3-319-67183-3_16 